\title{
Inelastic Interaction and Blowup New Solutions of Nonlinear and Dispersive Long Gravity Waves
}

\author{
Haiyong Qin $\left(\mathbb{D},{ }^{1,2}\right.$ Mostafa M. A. Khater $\mathbb{D}^{3,4}$ and Raghda A. M. Attia ${ }^{3,5}$ \\ ${ }^{1}$ School of Mathematics, Qilu Normal University, Jinan, 250200 Shandong Province, China \\ ${ }^{2}$ School of Control Science and Engineering, Shandong University, Jinan, 250061 Shandong, China \\ ${ }^{3}$ Department of Mathematics, Faculty of Science, Jiangsu University, China \\ ${ }^{4}$ Department of Mathematics, El Obour Institutes, Cairo, Egypt \\ ${ }^{5}$ Department of Basic Science, Higher Technological Institute 10th of Ramadan City, Egypt
}

Correspondence should be addressed to Haiyong Qin; qhymath@hotmail.com and Mostafa M. A. Khater; mostafa.khater2024@yahoo.com

Received 4 March 2020; Accepted 8 April 2020; Published 18 May 2020

Guest Editor: Chuanjun Chen

Copyright (c) 2020 Haiyong Qin et al. This is an open access article distributed under the Creative Commons Attribution License, which permits unrestricted use, distribution, and reproduction in any medium, provided the original work is properly cited.

\begin{abstract}
In this paper, the fractional Broer-Kaup (BK) system is investigated by studying its novel computational wave solutions. These solutions are constructed by applying two recent analytical schemes (modified Khater method and sech-tanh function expansion method). The BK system simulates the bidirectional propagation of long waves in shallow water. Moreover, it is used to study the interaction between nonlinear and dispersive long gravity waves. A new fractional operator is used to convert the fractional form of the BK system to a nonlinear ordinary differential system with an integer order. Many novel traveling wave solutions are constructed that do not exist earlier. These solutions are considered the icon key in the inelastic interaction of slow ions and atoms, where they were able to explain the physical nature of the nuclear and electronic stopping processes. For more illustration, some attractive sketches are also depicted for the interpretation physically of the achieved solutions.
\end{abstract}

\section{Introduction}

Studying the energetic atomic projectiles (atoms or ions) is one of the most exciting recent fields which has impacts on surface physics, plasma, and some related applications [13].13;3]. Moreover, it has an essential role which multicharged ions play as part of the solar wind in the space environment [4]. These applications include plasma-wall interaction in controlled thermonuclear fusion devices [5], surface analysis [6], single-particle detection [7], and electrical discharges [8]. The projectile energy deposition is connected with the intensity and nature of ion (atom) surface. Thus, both the nature and intensity of the ion depend on the potential and kinetic energies carried by a projectile toward the surface [9]. The energetic ion that can penetrate a solid surface is able to transfer its kinetic energy to target electrons, leading to atomic excitation, collective electron excitations, or ionization [10].
Partial differential equations (PDEs) have been playing an essential role in the energetic atomic projectiles where many nonlinear evolution equations have been derived to describe the dynamical behaviour of several phenomena in atomic and nuclear physics. Thus, partial differential equations (PDEs) have been playing an important role in the emerging technologies where many nonlinear evolution equations have been derived to describe the dynamical behaviour of some distinct phenomena, for example, nonlinear optics, fluid dynamics, Bose-Einstein condensates, and quantum mechanicss. However, the inadequacy of the PDEs with an integer order has been clarified because of the nonlocal property where this kind of equation does not explain that kind of properties. Therefore, several nature phenomena have been formulated with nonlinear PDEs with fractional order [11-35]. Thus, many fractional operators have been derived such as conformable fractional derivative, fractional Riemann-Liouville derivatives, Caputo, Caputo-Fabrizio definition, and so on [36-40]. 
These definitions have been employed to convert the fractional nonlinear partial differential equations to a nonlinear integer-order ordinary differential equation. Then, the computational and numerical schemes can be applied to get various types of solutions for these models and the examples of these schemes.

The $\mathscr{A} \mathscr{B} \mathscr{R}$ fractional operator is considered one of the most general recent fractional operators that is derived from avoiding the deficiencies and defects of some other fractional operator. This operator is defined as follows.

Definition 1. It is given by [41-44]

$$
\mathscr{A} \mathscr{B} \mathscr{R}^{\alpha} D_{a+} f(t)=\frac{B(\alpha)}{1-\alpha} \frac{d}{d t} \int_{a}^{t} f(x) g_{\alpha}\left(\frac{-\alpha(t-\alpha)^{\alpha}}{1-\alpha}\right) d x,
$$

where $g_{\alpha}$ stands for the Mittag-Leffler function, given by $[45,46]$

$$
g_{\alpha}\left(\frac{-\alpha(t-\alpha)^{\alpha}}{1-\alpha}\right)=\sum_{n=0}^{\infty} \frac{(-\alpha / 1-\alpha)^{n}(t-x)^{\alpha n}}{\Gamma(\alpha n+1)}
$$

and $B(\alpha)$ being a normalization function. Thus,

$$
\mathscr{A} \mathscr{B} \mathscr{R} D_{a+}^{\alpha} f(x)=\frac{B(\alpha)}{1-\alpha} \sum_{n=0}^{\infty}\left(\frac{-\alpha}{1-\alpha}\right)^{n R L} \mathscr{I}_{a}^{\alpha n} f(x)
$$

This research paper studies the fractional BK system that is given by [47-49].

$$
\left\{\begin{array}{l}
\mathscr{D}_{t}^{\alpha} \mathcal{S}=\frac{1}{2}\left(\mathcal{S}^{2}+2 \mathscr{W}-\mathcal{S}_{x}\right)_{x}, \\
\mathscr{D}_{t}^{\alpha} \mathscr{W}=\left(\mathcal{S} \mathscr{W}+\frac{1}{2} \mathscr{W}_{x}\right)_{x},
\end{array}\right.
$$

where $[(0<\alpha<1), \mathcal{S}=\mathcal{S}(x, t), \mathscr{W}=\mathscr{W}(x, t)]$ is unknown functions in time $(t)$ and place $(x)$. System (3) is used to simulate the bidirectional propagation of long waves in shallow water. Moreover, it studies the interaction between dispersive and nonlinear long gravity waves. System (3) is an integrable system and is related to the classical Boussinesq equation that is given by [50]

$$
\mathscr{B}_{t t}-\lambda^{2} \mathscr{B}_{x x}-\beta \mathscr{B}_{x x x x}-\alpha\left(\mathscr{B}^{2}\right)_{x x}=0
$$

where $[\lambda, \alpha, \beta]$ are arbitrary constants. Equation (5) is a model of nonlinear dispersive waves. Employing the next wave transformation (the $\mathscr{A} \mathscr{B} \mathscr{R}$ fractional operator) $\left[\mathrm{Y}=\left(\left((1-\alpha)\left(c t^{-n \alpha}\right) / B(\alpha) \sum_{n=0}^{\infty}(-(\alpha /(1-\alpha)))^{n} \Gamma(1-\alpha n)\right)+\right.\right.$ $x), \delta=\delta(Y), \mathscr{W}=\mathscr{W}(\mathrm{Y})$, where $(c)$ is an arbitrary constant] to the system $((3))$; then, differentiate the results once and substitute the second equation of the system into the first equation leading to convert the system into the next equation

$$
4 c^{2} \mathcal{S}-6 c \mathcal{S}^{2}+2 \mathcal{S}^{3}-\mathcal{S}^{\prime \prime}=0
$$

Applying the homogeneous balance principle to Equation (6), yields $(m=1)$.

The rest of research paper is organized as follows: Section 2 applies the modified Khater method and sech-tanh functions expansion method to the suggested model to get novel solitary wave solutions of it. Section 3 explains the physical interpretation of the shown sketches in our paper. Section 4 shows the novelty of our paper by comparing our results with those obtained in previous research papers. Section 5 explains the conclusion of all the steps of our paper in detail.

\section{Application}

Here, in this section, the modified Khater method and sechtanh functions expansion method are applied to the $\mathscr{B} \mathscr{K}$ equation to simulate the bi-directional propagation of long waves in sha llow water.

2.1. The Modified Khater Method. Applying the modified Khater method to Equation (6) leads to formulate the general solution of this model in the following formula

$$
\begin{aligned}
\mathcal{S}(\mathrm{Y}) & =\sum_{i=1}^{m} a_{i} \mathscr{K}^{i \mathscr{F}(\mathrm{Y})}+\sum_{i=1}^{m} b_{i} \mathscr{K}^{-i \mathscr{F}(\mathrm{Y})}+a_{0} \\
& =a_{1} \mathscr{K}^{\mathscr{F}(\mathrm{Y})}+a_{0}+b_{1} \mathscr{K}^{-\mathscr{F}(\mathrm{Y})}
\end{aligned}
$$

where $\left[a_{0}, a_{1}, b_{1}\right]$ are arbitrary constants to be determined later. Additionally, $\mathscr{F}(\mathrm{Y})$ is the solution function of the following ordinary differential equation

$$
\mathscr{F}^{\prime}(\mathrm{Y})=\frac{1}{\ln (\mathscr{K})}\left[\delta \mathscr{K}^{\mathscr{F}(\mathrm{Y})}+\mathrm{Q} \mathscr{K}^{-\mathscr{F}(\mathrm{Y})}+\chi\right]
$$

where $[\delta, \varrho, \chi]$ are arbitrary constants. Substituting Equation (7) along (7) into Equation (6) and collecting all terms with the same power of $\left[\mathscr{K}^{i \mathscr{F}(\mathrm{Y})}, i=-5,-4, \cdots, 4,5\right]$, give a system of the algebraic equation. Using the Mathematica 12 program for solving this system, yields

Family 2.

$$
\begin{gathered}
{\left[a_{0} \longrightarrow \frac{1}{2}\left(\sqrt{\chi^{2}-4 \delta \mathrm{Q}}+\chi\right), a_{1} \longrightarrow \delta, b_{1}\right.} \\
\left.\longrightarrow 0, c \longrightarrow \frac{1}{2} \sqrt{\chi^{2}-4 \delta \mathrm{\varrho}}\right] .
\end{gathered}
$$

Thus, the explicit wave solutions of Equation (4) are formulated in the following formulas 
For $\left[\chi^{2}-4 \delta \mathrm{Q}>0 \& \delta \neq 0\right]$,

$$
\begin{aligned}
\mathcal{S}_{1}(x, t)= & -\frac{1}{2} \sqrt{\chi^{2}-4 \delta \mathrm{\varrho}}\left(\operatorname { t a n h } \left(\frac{1}{2} x \sqrt{\chi^{2}-4 \delta \mathrm{\varrho}}\right.\right. \\
& \left.\left.-\frac{(\alpha-1) t^{-\alpha}\left(\chi^{2}-4 \delta \mathrm{\varrho}\right)}{4 B(\alpha) \sum_{n=0}^{\infty}(-\alpha /(\alpha /(1-\alpha)))^{n} \Gamma(1-\alpha n)}\right)-1\right),
\end{aligned}
$$

$$
\begin{aligned}
\mathcal{S}_{2}(x, t)= & -\frac{1}{2} \sqrt{\chi^{2}-4 \delta \mathrm{Q}}\left(\operatorname { c o t h } \left(\frac{1}{2} x \sqrt{\chi^{2}-4 \delta \mathrm{\varrho}}\right.\right. \\
& \left.\left.-\frac{(\alpha-1) t^{-\alpha}\left(\chi^{2}-4 \delta \mathrm{\varrho}\right)}{4 B(\alpha) \sum_{n=0}^{\infty}(-(\alpha /(1-\alpha)))^{n} \Gamma(1-\alpha n)}\right)-1\right) .
\end{aligned}
$$

For $[\delta \mathrm{Q}<0 \& \varrho \neq 0 \& \delta \neq 0 \& \chi=0]$,

$$
\begin{aligned}
& \mathcal{S}_{3}(x, t)=-\sqrt{-\delta \mathrm{\varrho}}\left(\tanh \left(\frac{(\alpha-1) \delta \mathrm{\varrho} t^{-\alpha}}{B(\alpha) \sum_{n=0}^{\infty}(-(\alpha /(1-\alpha)))^{n} \Gamma(1-\alpha n)}+x \sqrt{-\delta \mathrm{\varrho}}\right)-1\right) \\
& \mathcal{S}_{4}(x, t)=-\sqrt{-\delta \mathrm{\varrho}}\left(\operatorname{coth}\left(\frac{(\alpha-1) \delta \mathrm{\varrho} t^{-\alpha}}{B(\alpha) \sum_{n=0}^{\infty}(-(\alpha /(1-\alpha)))^{n} \Gamma(1-\alpha n)}+x \sqrt{-\delta \mathrm{\varrho}}\right)-1\right) .
\end{aligned}
$$

For $[\chi=0 \& Q=-\delta]$,

$$
\begin{aligned}
\mathcal{S}_{5}(x, t)= & \sqrt{\mathrm{Q}^{2}}-\mathrm{\varrho} \operatorname{coth}(\mathrm{\varrho}(x \\
& \left.\left.-\frac{(\alpha-1) \sqrt{\mathrm{\varrho}^{2}} t^{-\alpha}}{B(\alpha) \sum_{n=0}^{\infty}(-(\alpha /(1-\alpha)))^{n} \Gamma(1-\alpha n)}\right)\right) .
\end{aligned}
$$

For $[\chi=\delta=\kappa \& Q=0]$,

$$
\begin{aligned}
\mathcal{S}_{6}(x, t)= & \frac{1}{2}\left(\sqrt{\kappa^{2}}-\kappa \operatorname{coth}\left(\frac{1}{2} \kappa(x\right.\right. \\
& \left.\left.\left.-\frac{(\alpha-1) \sqrt{\kappa^{2}} t^{-\alpha}}{2 B(\alpha) \sum_{n=0}^{\infty}(-(\alpha /(1-\alpha)))^{n} \Gamma(1-\alpha n)}\right)\right)\right) .
\end{aligned}
$$

For $[\varrho=0 \& \chi \neq 0 \& \delta \neq 0]$

$$
\mathcal{S}_{7}(x, t)=\frac{1}{2}\left(\chi\left(-\frac{4}{\delta \exp \left(\chi\left(x-(\alpha-1) \sqrt{\chi^{2}} t^{-\alpha} / 2 B(\alpha) \sum_{n=0}^{\infty}(-(\alpha /(1-\alpha)))^{n} \Gamma(1-\alpha n)\right)\right)-2}-1\right)+\sqrt{\chi^{2}}\right)
$$

Family 3.

$$
\begin{gathered}
{\left[a_{0} \longrightarrow \frac{1}{2}\left(\sqrt{\chi^{2}-4 \delta \rho}+\chi\right), a_{1} \longrightarrow 0, b_{1}\right.} \\
\left.\longrightarrow \rho, c \longrightarrow \frac{1}{2} \sqrt{\chi^{2}-4 \delta \rho}\right]
\end{gathered}
$$

Thus, the explicit wave solutions of Equation (4) are formulated in the following formulas

For $\left[\chi^{2}-4 \delta \mathrm{\varrho}>0 \& \delta \neq 0\right]$,

$$
\begin{aligned}
& \delta_{8}(x, t)=\frac{1}{2}\left(-\frac{4 \delta \mathrm{\varrho}}{\sqrt{\chi^{2}-4 \delta \mathrm{\varrho}} \tanh \left(1 / 2 x \sqrt{\chi^{2}-4 \delta \mathrm{\varrho}}-(\alpha-1) t^{-\alpha}\left(\chi^{2}-4 \delta \mathrm{\varrho}\right) / 4 B(\alpha) \sum_{n=0}^{\infty}(-(\alpha /(1-\alpha)))^{n} \Gamma(1-\alpha n)\right)+\chi}+\sqrt{\chi^{2}-4 \delta \varrho}+\chi\right) \\
& \delta_{9}(x, t)=\frac{1}{2}\left(-\frac{4 \delta \mathrm{\varrho}}{\sqrt{\chi^{2}-4 \delta \varrho} \operatorname{coth}\left(1 / 2 x \sqrt{\chi^{2}-4 \delta \varrho}-(\alpha-1) t^{-\alpha}\left(\chi^{2}-4 \delta \varrho\right) / 4 B(\alpha) \sum_{n=0}^{\infty}(-(\alpha /(1-\alpha)))^{n} \Gamma(1-\alpha n)\right)+\chi}+\sqrt{\chi^{2}-4 \delta \varrho}+\chi\right) .
\end{aligned}
$$


For $[\delta \varrho<0 \& \varrho \neq 0 \& \delta \neq 0 \& \chi=0]$,

$$
\begin{aligned}
& \mathcal{S}_{10}(x, t)=\sqrt{-\delta \mathrm{\varrho}}\left(\operatorname{coth}\left(\frac{(\alpha-1) \delta \mathrm{\varrho} t^{-\alpha}}{B(\alpha) \sum_{n=0}^{\infty}(-(\alpha /(1-\alpha)))^{n} \Gamma(1-\alpha n)}+x \sqrt{-\delta \mathrm{\varrho}}\right)+1\right), \\
& \mathcal{S}_{11}(x, t)=\sqrt{-\delta \mathrm{\varrho}}\left(\tanh \left(\frac{(\alpha-1) \delta \mathrm{\varrho} t^{-\alpha}}{B(\alpha) \sum_{n=0}^{\infty}(-(\alpha /(1-\alpha)))^{n} \Gamma(1-\alpha n)}+x \sqrt{-\delta \mathrm{\varrho}}\right)+1\right) .
\end{aligned}
$$

For $[\chi=0 \& \varrho=-\delta]$,

$$
\mathcal{S}_{12}(x, t)=\varrho \tanh \left(\rho\left(x-\frac{(\alpha-1) \sqrt{\mathrm{Q}^{2}} t^{-\alpha}}{B(\alpha) \sum_{n=0}^{\infty}(-(\alpha /(1-\alpha)))^{n} \Gamma(1-\alpha n)}\right)\right)+\sqrt{\mathrm{\varrho}^{2}} .
$$

For $[\chi=\mathrm{Q} / 2=\kappa \& \delta=0]$,

$$
\mathcal{S}_{13}(x, t)=\frac{1}{2}\left(\frac{4 \kappa}{\exp \left(\kappa\left(x-(\alpha-1) \sqrt{\kappa^{2}} t^{-\alpha} / 2 B(\alpha) \sum_{n=0}^{\infty}(-(\alpha /(1-\alpha)))^{n} \Gamma(1-\alpha n)\right)\right)-2}+\sqrt{\kappa^{2}}+\kappa\right) .
$$

For $[\delta=0 \& \chi \neq 0 \& \varrho \neq 0]$,

$$
\mathcal{S}_{14}(x, t)=\frac{1}{2}\left(-\frac{2 \chi \varrho}{\varrho-\chi \exp \left(\chi\left(x-(\alpha-1) \sqrt{\chi^{2}} t^{-\alpha} / 2 B(\alpha) \sum_{n=0}^{\infty}(-(\alpha /(1-\alpha)))^{n} \Gamma(1-\alpha n)\right)\right)}+\sqrt{\chi^{2}}+\chi\right)
$$

2.2. The Sech-Tanh Function Expansion Method. Applying the sech-tanh function expansion method to Equation (6) leads to formulate the general solution of this model in the following formula:

$$
\begin{aligned}
\mathcal{S}(\mathrm{Y}) & =\sum_{i=1}^{m} \operatorname{sech}^{i-1}(\mathrm{Y})\left(a_{i} \operatorname{sech}(\mathrm{Y})+b_{i} \tanh (\mathrm{Y})\right)+a_{0} \\
& =a_{1} \operatorname{sech}(\mathrm{Y})+a_{0}+b_{1} \tanh (\mathrm{Y}),
\end{aligned}
$$

where $\left[a_{0}, a_{1}, b_{1}\right]$ are arbitrary constants to be determined later. Substituting Equation (23) into Equation (6) and collecting all terms with the same power of $[\operatorname{sech}(\mathrm{Y}), \tanh (\mathrm{Y})$ ], give a system of the algebraic equation. Using the Mathematica 12 program for solving this system, yields
Family 4.

$$
\left[a_{0} \longrightarrow-1, a_{1} \longrightarrow 0, b_{1} \longrightarrow 1, c \longrightarrow-1\right]
$$

Thus, the explicit wave solutions of Equation (4) are formulated in the following formulas:

$\mathcal{S}_{15}(x, t)=\tanh \left(\frac{(\alpha-1) t^{-\alpha}}{B(\alpha) \sum_{n=0}^{\infty}(-(\alpha /(1-\alpha)))^{n} \Gamma(1-\alpha n)}+x\right)-1$

Family 5.

$$
\left[a_{0} \longrightarrow 1, a_{1} \longrightarrow 0, b_{1} \longrightarrow 1, c \longrightarrow 1\right]
$$




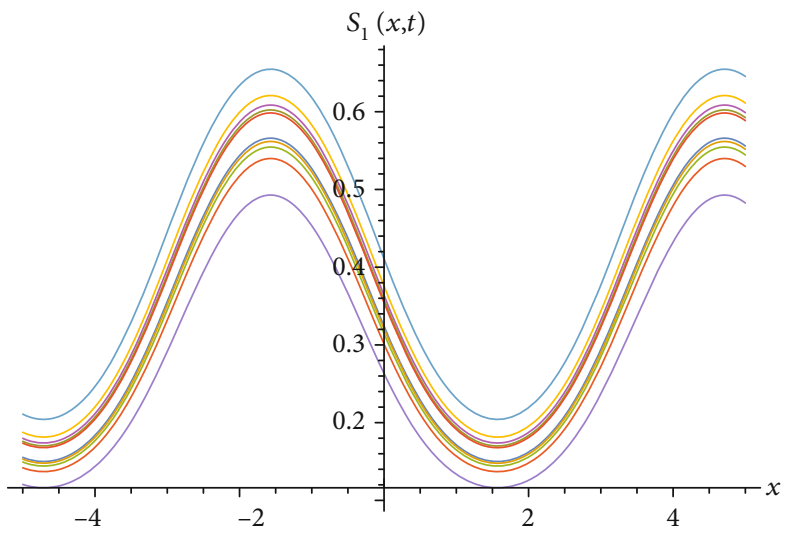

(a)

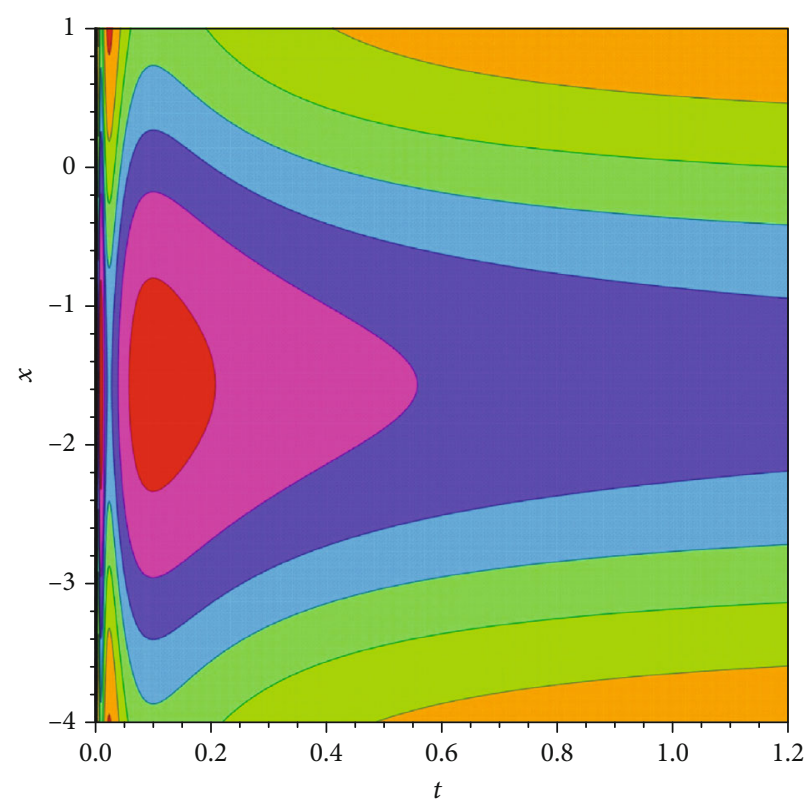

(b)

Figure 1: Numerical simulation of Equation (10) in two-dimensional and contour sketches.

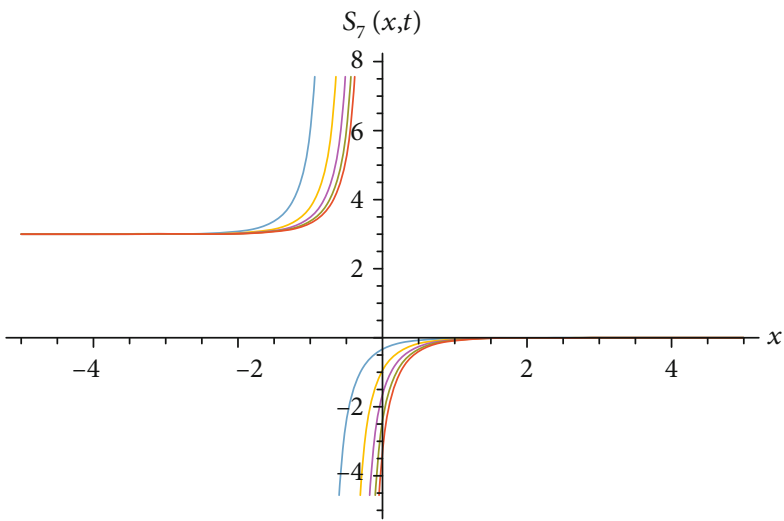

(a)

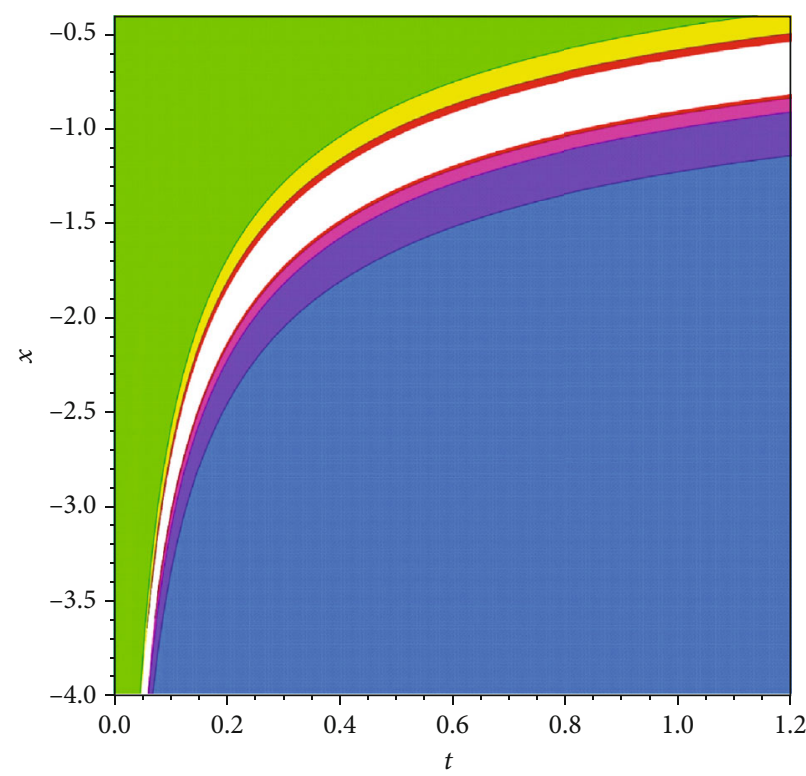

(b)

FIGURE 2: Numerical simulation of Equation (15) in two-dimensional and contour sketches.

Thus, the explicit wave solutions of Equation (4) are formulated in the following formulas:

$\mathcal{S}_{16}(x, t)=\tanh \left(x-\frac{(\alpha-1) t^{-\alpha}}{B(\alpha) \sum_{n=0}^{\infty}(-(\alpha /(1-\alpha)))^{n} \Gamma(1-\alpha n)}\right)+1$.

\section{Figure Interpretation}

This section gives the physical interpretation of the shown figures in our paper. All our obtained solutions are consid- ered traveling wave solutions. In the following lines, we give the physical interpretation of the shown figures:

(1) Figure 1 shows the bell wave solution (8) in the twodimensional plot (a) to explain the wave propagation pattern of the wave along the $x$-axis and the contour plot (b) to explain the overhead view of the solution when $[\alpha=(1 / 2), \delta=1, \chi=3, \varrho=2$.]

(2) Figure 2 shows the singular wave solution (13) in the two-dimensional plot (a) to explain the wave propagation pattern of the wave along the $x$-axis and the 


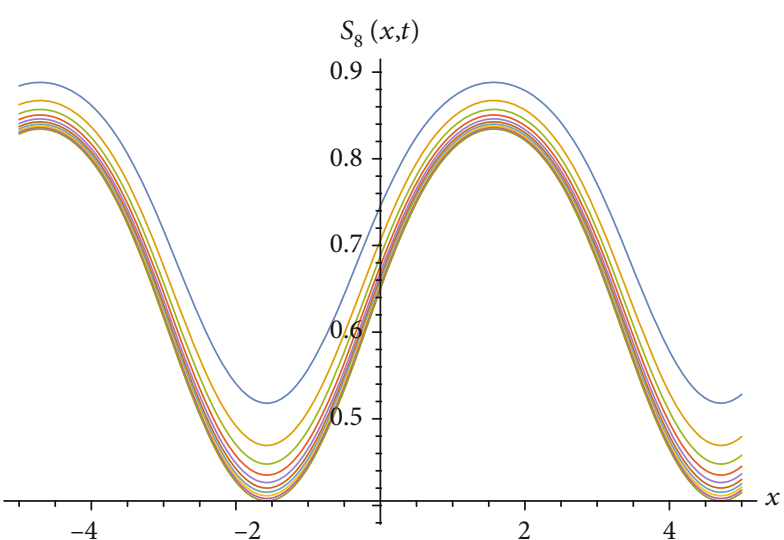

(a)

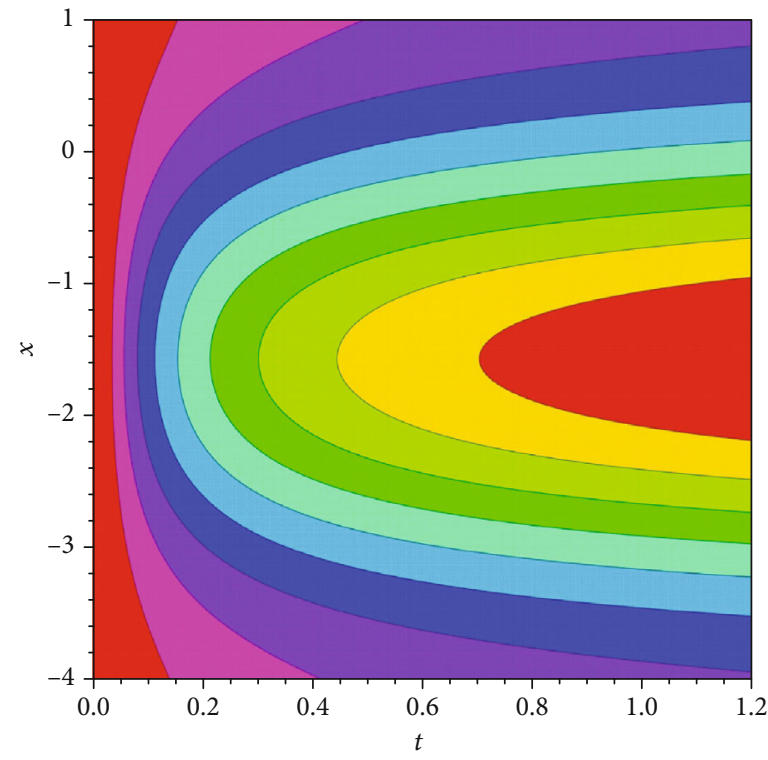

(b)

Figure 3: Numerical simulation of Equation (17) in two-dimensional and contour sketches.

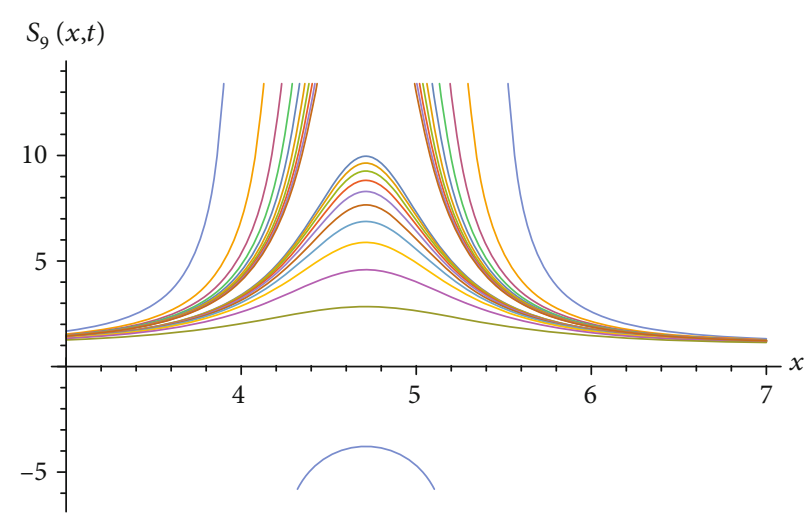

(a)

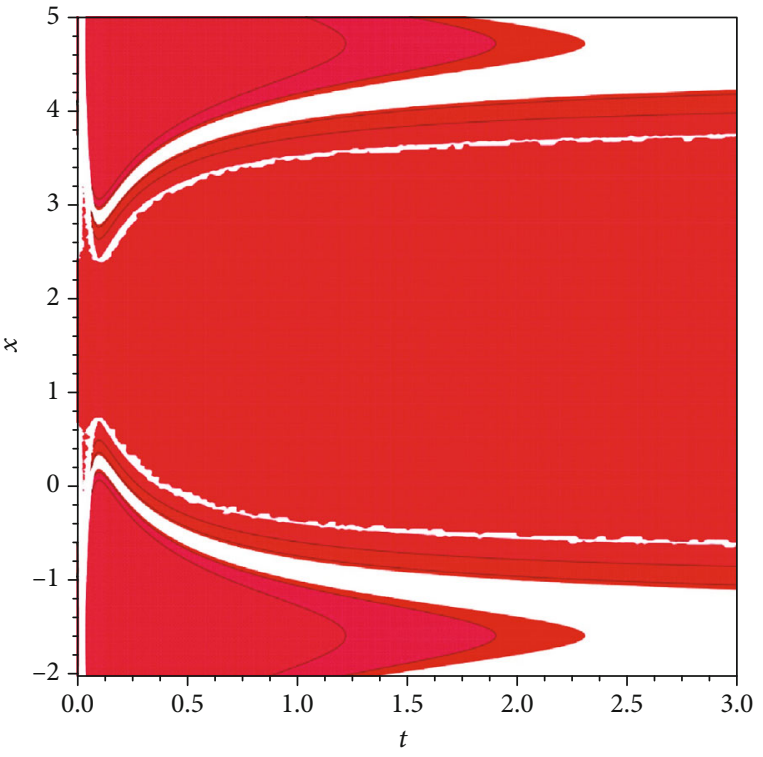

(b)

Figure 4: Numerical simulation of Equation (18) in two-dimensional and contour sketches.

contour plot (b) to explain the overhead view of the solution when $[\alpha=(1 / 2), \delta=1, \chi=3$.]

(3) Figure 3 shows the W-wave solution (14) in the twodimensional plot (a) to explain the wave propagation pattern of the wave along the $x$-axis and the contour plot (b) to explain the overhead view of the solution when $[\alpha=(1 / 2), \delta=1, \chi=5, \mathrm{Q}=6$.

(4) Figure 4 shows the rouge-wave solution (15) in the two-dimensional plot (a) to explain the wave propagation pattern of the wave along the $x$-axis and the contour plot (b) to explain the overhead view of the solution when $[\alpha=(1 / 2), \delta=1, \chi=5, \varrho=6$.]

(5) Figure 5 shows the Breath-wave solution (21) in the two-dimensional plot (a) to explain the wave propagation pattern of the wave along the $x$-axis and the contour plot (b) to explain the overhead view of the solution when $[\alpha=(1 / 2)]$.

(6) Figure 6 shows the kink-wave solution (22) in the two-dimensional plot (a) to explain the wave propagation pattern of the wave along the $x$-axis and the 


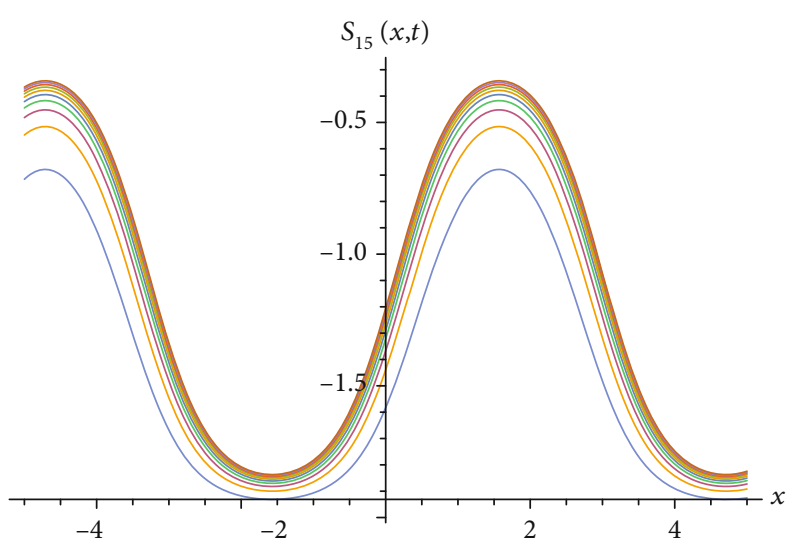

(a)

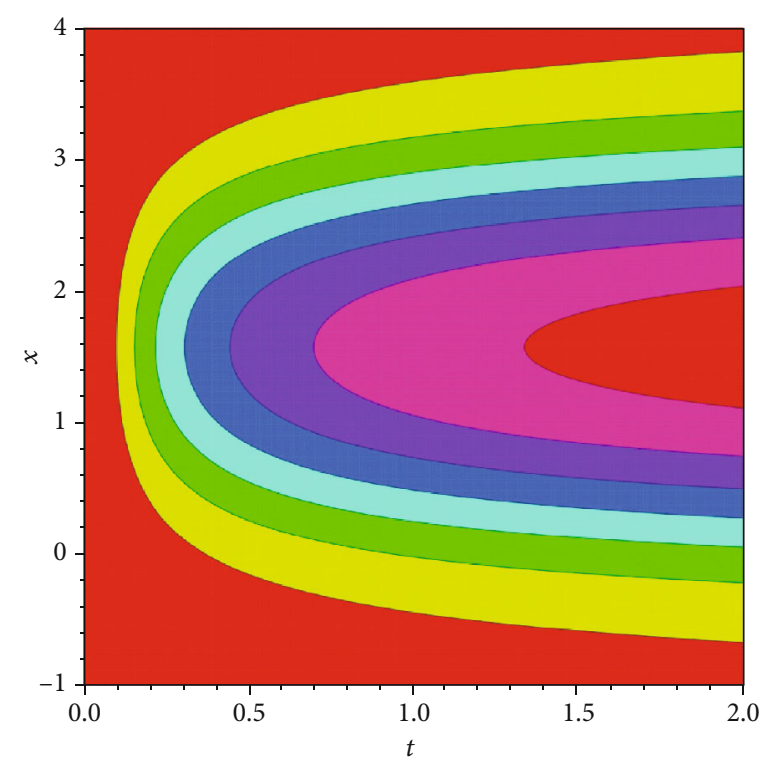

(b)

Figure 5: Numerical simulation of Equation (25) in two-dimensional and contour sketches.

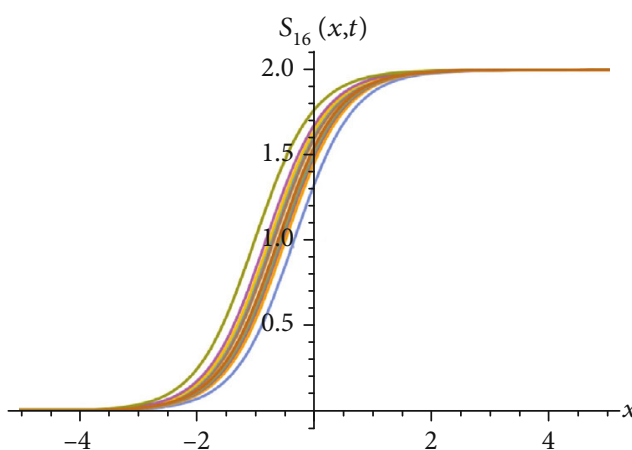

(a)

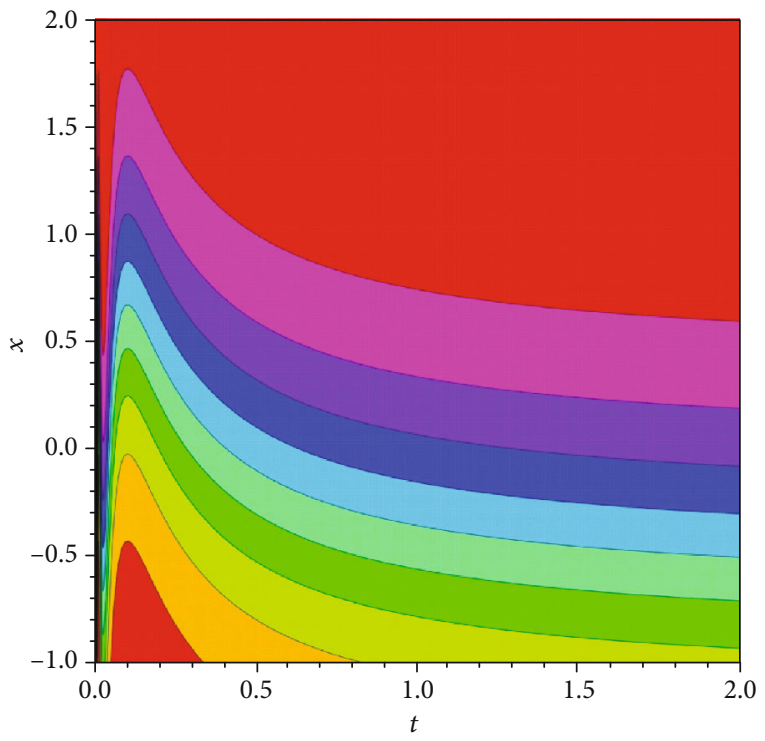

(b)

FIGURE 6: Numerical simulation of Equation (27) in two-dimensional and contour sketches.

contour plot (b) to explain the overhead view of the solution when $[\alpha=(1 / 2)]$.

\section{Results and Discussion}

This section shows the novelty of this research paper by explaining the comparison between our obtained solutions and those obtained in previous paper.

4.1. Computational Schemes. This paper investigated the analytical solutions of the fractional BK system by using two recent computational schemes (modified Khater method and sech-tanh function expansion method). These methods are considered recent analytical schemes in this field, and they were not applied to this model yet.

\subsection{Obtained Computational Wave Solutions}

(i) Equation (10) is equal to Equation (25) when $\left[\chi^{2}-\right.$ $4 \mathrm{\varrho} \delta=4]$

(ii) All our obtained solutions are different from those obtained in [47-49] where the authors of [47-49] applied different methods to solve the BK system with an integer order. On the other hand, we investigated the fractional form of this model 


\section{Conclusion}

In this paper, we investigated new soliton wave solutions of the nonlinear fractional BK system by using two recent analytical schemes. A new fractional operator is used to convert the fractional form of this model to a nonlinear partial differential equation with an integer order. The modified Khater method and sech-tanh function expansion method were applied to this model. Some new soliton wave solutions were obtained, and some of them were explained by plotting them in two-dimensional and contour plots of these solutions. The novelty of our paper was shown by making the comparison between our obtained solutions and that was purchased in previously published articles.

\section{Data Availability}

The data that support the findings of this study are available from the corresponding author upon reasonable request.

\section{Ethical Approval}

The authors state that this research complies with ethical standards. This research does not involve either human participants or animals.

\section{Conflicts of Interest}

The authors declare that there is no conflict of interests regarding the publication of this article.

\section{Acknowledgments}

This research is supported by the National Natural Science Foundation of China, (Grant Nos. 61973193 and 61807010), Shandong Provincial Natural Science Foundation (Grant Nos. ZR2017BA009, ZR2018MA009, and ZR2016AB04), a Project of Shandong Province Higher Educational Science and Technology Program (Grant No. J17KB121), Foundation for Young Teachers of Qilu Normal University (Grant Nos. 2016L0605, 2015L0603, 2017JX2311, and 2017JX2312).

\section{References}

[1] A. Mertens and H. Winter, "Energy transfer from fast atomic projectiles to a crystal lattice under channeling conditions," Physical review letters, vol. 85, no. 13, pp. 2825-2828, 2000.

[2] S. Suarez, R. O. Barrachina, and W. Meckbach, "Double scattering effects in the ionization spectrum produced by single energetic atomic collisions," Physical review letters, vol. 77, no. 3, pp. 474-477, 1996.

[3] A. Wucher, L. Breuer, and N. Winograd, "Ionization probability of sputtered indium under irradiation with $20 \mathrm{-keV}$ fullerene and argon gas cluster projectiles," International Journal of Mass Spectrometry, vol. 438, pp. 13-21, 2019.

[4] G. C. Demontis, M. M. Germani, E. G. Caiani, I. Barravecchia, C. Passino, and D. Angeloni, "Human pathophysiological adaptations to the space environment," Frontiers in physiology, vol. 8, p. 547, 2017.

[5] M. Rubel, P. Petersson, E. Alves et al., "The role and application of ion beam analysis for studies of plasma-facing compo- nents in controlled fusion devices," Nuclear Instruments and Methods in Physics Research Section B, vol. 371, pp. 4-11, 2016.

[6] S.-Y. Ding, J. Yi, J.-F. Li et al., "Nanostructure-based plasmonenhanced raman spectroscopy for surface analysis of materials," Nature Reviews Materials, vol. 1, no. 6, pp. 1-16, 2016.

[7] E. Davis, G. Bentsen, and M. Schleier-Smith, "Approaching the Heisenberg limit without single-particle detection," Physical review letters, vol. 116, no. 5, article 053601, 2016.

[8] M. A. Malik, A. Ghaffar, and S. A. Malik, "Water purification by electrical discharges," Plasma Sources Science and Technology, vol. 10, no. 1, pp. 82-91, 2001.

[9] M. F. Russo and B. J. Garrison, "Mesoscale energy deposition footprint model for kiloelectronvolt cluster bombardment of solids," Analytical Chemistry, vol. 78, no. 20, pp. 7206-7210, 2006.

[10] B. H. Mauk, J. B. Blake, D. N. Baker et al., "The energetic particle detector (EPD) investigation and the energetic ion spectrometer (EIS) for the magnetospheric multiscale (MMS) mission," Space Science Reviews, vol. 199, no. 1-4, pp. 471514, 2016.

[11] T. A. Ahmad, M. M. A. Khater, R. A. M. Attia, A.-H. AbdelAty, and D. Lu, "Abundant numerical and analytical solutions of the generalized formula of Hirota-Satsuma coupled KdV system," Chaos, Solitons \& Fractals, vol. 131, article 109473, 2020.

[12] R. A. M. Attia, D. Lu, T. Ak, and M. M. A. Khater, "Optical wave solutions of the higher-order nonlinear Schrödinger equation with the non-Kerr nonlinear term via modified Khater method," Modern Physics Letters B, vol. 34, no. 5, article 2050044, 2020.

[13] R. Attia, D. Lu, and M. M. A. Khater, "Chaos and relativistic energy-momentum of the nonlinear time fractional Duffing equation," Mathematical and Computational Applications, vol. 24, no. 1, p. 10, 2019.

[14] C. Chen, K. Li, Y. Chen, and Y. Huang, "Two-grid finite element methods combined with Crank-Nicolson scheme for nonlinear Sobolev equations," Advances in Computational Mathematics, vol. 45, no. 2, pp. 611-630, 2019.

[15] C. Chen, H. Liu, X. Zheng, and H. Wang, "A two-grid MMOC finite element method for nonlinear variable-order timefractional mobile/immobile advection-diffusion equations," Computers \& Mathematics with Applications, vol. 79, no. 9, pp. 2771-2783, 2020.

[16] M. Eslami, F. S. Khodadad, F. Nazari, and H. Rezazadeh, "The first integral method applied to the Bogoyavlenskii equations by means of conformable fractional derivative," Optical and Quantum Electronics, vol. 49, no. 12, p. 391, 2017.

[17] M. Eslami and H. Rezazadeh, "The first integral method for $\mathrm{Wu}$-Zhang system with conformable time-fractional derivative," Calcolo, vol. 53, no. 3, pp. 475-485, 2016.

[18] M. M. A. Khater, R. A. M. Attia, A.-H. Abdel-Aty, M. A. Abdou, H. Eleuch, and D. Lu, "Analytical and semianalytical ample solutions of the higher-order nonlinear Schrödinger equation with the non-Kerr nonlinear term," Results in Physics, vol. 16, article 103000, 2020.

[19] M. M. A. Khater, D. Lu, and R. A. M. Attia, "Dispersive long wave of nonlinear fractional $\mathrm{Wu}$-Zhang system via a modified auxiliary equation method," AIP Advances, vol. 9, no. 2, article 025003, 2019.

[20] M. M. A. Khater, D. Lu, and R. A. M. Attia, "Lump soliton wave solutions for the $(2+1)$-dimensional Konopelchenko- 
Dubrovsky equation and KdV equation," Modern Physics Letters B, vol. 33, no. 18, article 1950199, 2019.

[21] M. M. A. Khater, C. Park, A.-H. Abdel-Aty, R. A. M. Attia, and $\mathrm{D}$. Lu, "On new computational and numerical solutions of the modified Zakharov-Kuznetsov equation arising in electrical engineering," Alexandria Engineering Journal, vol. 2020, 2020.

[22] M. M. A. Khater, A. R. Seadawy, and D. Lu, "Elliptic and solitary wave solutions for Bogoyavlenskii equations system, couple Boiti-Leon-Pempinelli equations system and Timefractional Cahn-Allen equation," Results in physics, vol. 7, pp. 2325-2333, 2017.

[23] M. M. A. Khater, A. R. Seadawy, and D. Lu, "Dispersive optical soliton solutions for higher order nonlinear Sasa-Satsuma equation in mono mode fibers via new auxiliary equation method," Superlattices and Microstructures, vol. 113, pp. 346-358, 2018.

[24] F. S. Khodadad, F. Nazari, M. Eslami, and H. Rezazadeh, "Soliton solutions of the conformable fractional Zakharov-Kuznetsov equation with dual-power law nonlinearity," Optical and Quantum Electronics, vol. 49, no. 11, p. 384, 2017.

[25] D. Lu, A. R. Seadawy, and M. M. A. Khater, "Structures of exact and solitary optical solutions for the higher-order nonlinear Schrödinger equation and its applications in monomode optical fibers," Modern Physics Letters B, vol. 33, no. 23, article 1950279, 2019.

[26] W. Ma, C. Li, and J. Deng, "Synchronization in tempered fractional complex networks via auxiliary system approach," Complexity, vol. 2019, 12 pages, 2019.

[27] M. S. Osman, D. Lu, and M. M. A. Khater, "A study of optical wave propagation in the nonautonomous Schrödinger-Hirota equation with power-law nonlinearity," Results in Physics, vol. 13, article 102157, 2019.

[28] H. Rezazadeh, A. Korkmaz, M. M. A. Khater, M. Eslami, D. Lu, and R. A. M. Attia, "New exact traveling wave solutions of biological population model via the extended rational sinhcosh method and the modified Khater method," Modern Physics Letters B, vol. 33, no. 28, article 1950338, 2019.

[29] X. Zhang, J. Jiang, Y. Wu, and Y. Cui, "Existence and asymptotic properties of solutions for a nonlinear Schrödinger elliptic equation from geophysical fluid flows," Applied Mathematics Letters, vol. 90, pp. 229-237, 2019.

[30] X. Zhang, J. Jiang, Y. Wu, and Y. Cui, “The existence and nonexistence of entire large solutions for a quasilinear schrödinger elliptic system by dual approach," Applied Mathematics Letters, vol. 100, article 106018, 2020.

[31] X. Zhang, L. Liu, Y. Wu, and Y. Cui, "The existence and nonexistence of entire large solutions for a quasilinear Schrödinger elliptic system by dual approach," Journal of Mathematical Analysis and Applications, vol. 464, no. 2, pp. 1089-1106, 2018.

[32] X. Zhang, L. Liu, Y. Wu, and Y. Cui, "Existence of infinitely solutions for a modified nonlinear Schrödinger equation via dual approach," Electronic Journal of Differential Equations, vol. 147, pp. 1-15, 2018.

[33] X. Zhang, L. Liu, Y. Wu, and Y. Cui, "A sufficient and necessary condition of existence of blow-up radial solutions for a k-Hessian equation with a nonlinear operator," Nonlinear Analysis: Modelling and Control, vol. 25, pp. 126-143, 2020.

[34] X. Zhang, Y. Wu, and L. Caccetta, "Nonlocal fractional order differential equations with changing-sign singular perturba- tion," Applied Mathematical Modelling, vol. 39, no. 21, pp. 6543-6552, 2015.

[35] X. Zhang, J. Xu, J. Jiang, Y. Wu, and Y. Cui, "The convergence analysis and uniqueness of blow-up solutions for a dirichlet problem of the general k-Hessian equations," Applied Mathematics Letters, vol. 102, article 106124, 2020.

[36] T. Abdeljawad, "On conformable fractional calculus," Journal of Computational and Applied Mathematics, vol. 279, pp. 5766, 2015.

[37] A. Atangana and J. F. Gómez-Aguilar, "Numerical approximation of Riemann-Liouville definition of fractional derivative: from Riemann-Liouville to Atangana-Baleanu," Numerical Methods for Partial Differential Equations, vol. 34, no. 5, pp. 1502-1523, 2018.

[38] W. S. Chung, "Fractional newton mechanics with conformable fractional derivative," Journal of computational and applied mathematics, vol. 290, pp. 150-158, 2015.

[39] J. Hristov, "Derivatives with non-singular kernels from the Caputo-Fabrizio definition and beyond: appraising analysis with emphasis on diffusion models," Frontier Fractal Calculus, vol. 1, pp. 270-342, 2017.

[40] K. M. Owolabi and A. Atangana, "Numerical approximation of nonlinear fractional parabolic differential equations with Caputo-Fabrizio derivative in Riemann-Liouville sense," Chaos, Solitons \& Fractals, vol. 99, pp. 171-179, 2017.

[41] C. Park, M. M. A. Khater, R. A. M. Attia, W. Alharbi, and S. S. Alodhaibi, "An explicit plethora of solution for the fractional nonlinear model of the low-pass electrical transmission lines via Atangana-Baleanu derivative operator," Alexandria Engineering Journal, 2020.

[42] N. A. Sheikh, F. Ali, M. Saqib, I. Khan, and S. A. A. Jan, "A comparative study of Atangana-Baleanu and CaputoFabrizio fractional derivatives to the convective flow of a generalized casson fluid," The European Physical Journal Plus, vol. 132, no. 1, article 52, 2017.

[43] H. Tajadodi, "A numerical approach of fractional advectiondiffusion equation with Atangana-Baleanu derivative," Chaos, Solitons \& Fractals, vol. 130, article 109527, 2020.

[44] C. Yue, M. M. A. Khater, R. A. M. Attia, and D. Lu, "The plethora of explicit solutions of the fractional KS equation through liquid-gas bubbles mix under the thermodynamic conditions via Atangana-Baleanu derivative operator," Advances in Difference Equations, vol. 2020, no. 1, Article ID 62, 2020.

[45] E. Mittal, S. Joshi, and R. M. Pandey, "On euler type integrals involving extended Mittag-Leffler functions," Boletim da Sociedade Paranaense de Matemática, vol. 38, no. 2, pp. 123-132, 2020.

[46] I. O. Sarumi, K. M. Furati, and A. Q. M. Khaliq, "Highly Accurate Global Padé Approximations of Generalized MittagLeffler Function and Its Inverse," Journal of Scientific Computing, vol. 82, no. 2, p. 46, 2020.

[47] Y.-H. Wang, "Construction of rational solutions for the (2+1)dimensional Broer-Kaup system," Modern Physics Letters B, vol. 33, no. 30, article 1950377, 2019.

[48] P. Zhao, E. Fan, and L. Lin, "Quasiperiodic solutions of the Kadomtsev-Petviashvili equation via the multidimensional Baker-Akhiezer function generated by the Broer-Kaup hierarchy," Journal of Mathematical Analysis and Applications, vol. 435 , no. 1 , pp. 38-60, 2016. 
[49] X.-H. Zhao, B. Tian, Y.-J. Guo, and H.-M. Li, "Solitons interaction and integrability for a $(2+1)$-dimensional variablecoefficient Broer-Kaup system in water waves," Modern Physics Letters B, vol. 32, no. 8, article 1750268, 2018.

[50] T. S. Jang, "A new dispersion-relation preserving method for integrating the classical Boussinesq equation," Communications in Nonlinear Science and Numerical Simulation, vol. 43, pp. 118-138, 2017. 